\title{
INVESTIGACIONES
}

\section{Lectura crítica y pensamiento reflexivo en la práctica pedagógica de profesores de básica secundaria}

\author{
Critical reading and reflective thinking in the pedagogical practice \\ of secondary school teachers

\section{Álvarez Maestre Annie Julieth ${ }^{a}$, Miryan Teresa Mendoza Meza, Lida Mercedes Moreno Rozo ${ }^{a}$, Garavito Patiño José Joan ${ }^{b}$} \\ ${ }^{a}$ Universidad Simón Bolívar. \\ a.alvarez@unisimonbolivar.edu.co \\ ${ }^{b}$ Coordinador de lenguaje y procesos comunicativos en Universidad Simón Bolívar. \\ j.garavito@unisimonbolivar.edu.co
}

\begin{abstract}
El presente estudio de investigación estuvo orientado a interpretar los alcances que tiene la lectura crítica y el pensamiento reflexivo en la práctica pedagógica en profesores de básica secundaria. Se asume como tema principal de la investigación las prácticas pedagógicas de los docentes de asignaturas que han evidenciado un desempeño académico alto por parte de los estudiantes en los registros de resultados de las pruebas externas, y su relación con la lectura crítica y el pensamiento reflexivo. El objeto de estudio y la metodología planteada posibilitan la selección de una muestra no probabilística, con informantes claves, debido a la naturaleza de la investigación. Como resultados se obtuvo que las prácticas pedagógicas de los participantes están relacionadas al hábito lector y el pensamiento reflexivo.
\end{abstract}

Palabras claves: Pedagogía, lectura, pensamiento crítico, enseñanza.

\section{ABSTRACT}

The present research study was aimed at interpreting the scope of critical reading and reflexive thinking in the pedagogical practice of secondary school teachers. The pedagogical practices of subject teachers who have shown high academic performance on the part of students in the records of external test results, and their relationship with critical reading and reflective thinking are assumed as the main theme of the research. The object of study and the methodology proposed enable the selection of a non-probabilistic sample, with key informants, due to the nature of the research. As results, it was obtained that the pedagogical practices of the participants are related to the reading habit and reflective thinking.

Key words: Pedagogy, reading, critical thinking, teaching. 


\section{INTRODUCCIÓN}

La lectura crítica no es una práctica generalizada entre las personas, sin embargo, cada día es más importante su práctica consciente por parte de los profesionales de la enseñanza, esencialmente, que requieren acceder a los códigos y categorías del mundo de la información, pues la vida moderna se mueve al ritmo del conocimiento (Flórez Ochoa, 2013).

La lectura crítica y el pensamiento reflexivo son dos categorías conceptuales ligadas por nexos de causalidad (Carlino, 2006; Klooster, Steele \& Bloem, 2001). Y las prácticas pedagógicas de los docentes pueden estar asociadas con estos fenómenos, en mayor o menor grado dependiendo de los hábitos de los maestros en la asunción de sus dinámicas de trabajo en el aula y de su saber pedagógico (Zuluaga, 1999). Un lector crítico, necesariamente desarrolla un pensamiento reflexivo, y a su vez el lector ha de ser igualmente un sujeto que escribe, pues la escritura posibilita un mejor acercamiento al proceso de interpretación y construcción de comprensiones (Cassany, 2005, 2013, 2016). El maestro que lee y escribe tiene mejores condiciones para pensar y actuar críticamente como persona, como profesional y como ciudadano (Freire, 1989, 2010).

Los docentes que leen son modelos que inspiran una suerte de respeto especial que, tanto estudiantes como pares maestros, prodigan y procuran emular de alguna manera (Eagleton, 1998). Un maestro es un agente educativo que cumple una función social y cultural de intermediación entre lo que se sabe y lo que debe saberse (Avendaño, 2013; Carlino, 2006). El maestro, que es un intelectual que desarrolla una labor académica, se apropia de ese rol en la medida que interactúa con sus estudiantes, con sus pares educadores y establece una conexión permanente con la lectura y con la escritura (Barrios García, 2015). De hecho, el maestro es un agente lector que promueve dentro de su dinámica educativa, procesos de lectura y escritura en función del aprendizaje (Sáiz y Rivas, 2008; Guzmán y Escobedo, 2006).

Tovar (2009) considera que la promoción de la lectura y la escritura en la escuela requieren fundamentalmente de la experiencia y vocación lectora del maestro para conseguir éxito en ese propósito. El docente lector es un animador de lecturas que promueve el libro y la lectura por placer, desde sus convicciones e imaginarios (Granado \& Puig, 2014; Zuluaga, 1999). Es un agente mediador de lectura que establece un diálogo asertivo permanente con sus estudiantes, invitándoles al encuentro con el texto no solamente en el aula, sino en la biblioteca, en casa y en los espacios de ocio, como una forma de mantener actualizado el mensaje de la experiencia lectora en un ámbito de complicidad con el estudiante o su interlocutor (Jones e Idol, 1990).

La lectura crítica como hábito especial en un docente ha de ser significativa en función de los desempeños de los estudiantes (Klooster, Steele \& Bloem, 2001; Munita, 2016). No es una relación axiomática, pero sí hay una marcada tendencia de reflejo no solo en la manera como el maestro maneja su discurso pedagógico, sino en las intencionalidades y motivaciones que le incitan a realizar un diseño y planificación de su trabajo a partir de su experiencia como lector, como impulsado por esa marca cultural que le deja su trasegar por el mundo de la exploración literaria, científica y académica (Alderson, 2000; Cassany, 2016). Por lo tanto, un eje problematizante de esta investigación giró en torno de la identificación de esas prácticas pedagógicas que condujeran a posibilitar mejores encuentros con la lectura y el pensamiento reflexivo, a partir de las propias acciones docentes. Sin esta perspectiva autónoma y formadora, lo que se tendría sería una reproducción de estándares básicos de competencias suministrados por cualquier sujeto que se desempeñe en la labor docente (Campos, 2007; Parodi, 2003). 
El mundo de la lectura no es complejo, es fascinante debido a las implicaciones que tiene, a los llamados que hace el texto cada vez que se aborda. La lectura crítica se hace más concreta cuando el lector escribe (Dubois, 2011). De hecho, toda lectura crítica ha de implicar un proceso escritural como acción refleja de interpretación y de comprensión (Cassany, 2013). En este mismo sentido, se comprende la escritura como práctica ineludible en los procesos de animación y promoción de la lectura, considerando que en el afán por desarrollar programas de cultivo y apreciación de la lectura se exacerba el énfasis sobre este proceso en detrimento de la escritura (Moreno, 2005).

De hecho, la escritura, esta valiosa tecnología de la palabra es un valioso recurso que debe utilizarse racionalmente para acercarse a los libros. La escritura es la base de todos los procesos escolares, incluso a aquellos que les compete la articulación de los pensamientos de forma oral (Medina, 2006; Ong, 2011). En este aspecto también se destaca la importancia de la oralidad en toda comunicación verbal, como primera forma de expresión humana (Ong y Hartley, 2016).

De la misma manera, Saussure (1959) concebía la escritura como un complemento de la oralidad. Esta visión de la lengua no debe ser extraña al maestro que orienta procesos educativos con estudiantes de secundaria, sobre todo si su intencionalidad pedagógica es promover la lectura crítica, ya que se trabaja con una realidad abstracta que es la lengua. Es por esa naturaleza poco concreta del idioma, que es indispensable que haya comprensión sobre estas dimensiones del mismo, haciendo reflexionar al estudiante sobre la importancia de la escritura en el desarrollo de la lengua a través de la historia y los procesos comunicativos interhumanos (Ong, 2011).

El docente lector toma conciencia de su rol, posee una formación literaria y disciplinar que lo ubica en un plano de compromiso con su quehacer, y desde esta perspectiva aborda las prácticas de aula en concordancia con sus anhelos de hallar en sus estudiantes un valioso auditorio para compartir experiencias y encuentros con el mundo (Kaufman, 1994; Munita, 2016). El rol del maestro es incitar a sus estudiantes a transformar su mundo. El mundo al que se hace referencia inicia con el encuentro de aula y no está enmarcado en su exclusividad porque de lo que se trata es de incitar a mayores reflexiones y pensamientos desde la reciprocidad de la lectura crítica (Rodríguez Castro, 2014).

Uno de los retos que deberá afrontar el docente será la construcción de textos, su aporte a la escritura no solo de él sino de aquellos a quienes llega (Caldera de Briceño, Escalante de Urrecheaga \& Terán de Serrentino, 2010). En este sentido, Tovar (2009), resalta entre otras muchas situaciones, la necesidad de ser buen lector y escritor para poder promover estos procesos en los estudiantes; el dilema estaría en torno a constatar esta realidad, ya que existen maestros lectores y escritores cuyas prácticas pedagógicas están muy lejos de obtener buenos resultados académicos entre sus estudiantes. El problema entonces habría que plantearlo desde la metodología (Garavito, 2016; Giroux, 1990).

Dubois (2011) manifiesta la importancia de entender que la formación de un docente en cualquier disciplina, aunque principalmente para el docente encargado de orientar procesos de lectura y escritura, debe abarcar necesariamente el saber, el hacer y el ser. Es de vital importancia en el proceso de formación de estudiantes hacia la consolidación del pensamiento reflexivo cimentado en la lectura crítica (Boisvert, 2004; Chois, 2005), que el docente sea un lector crítico, un ser humano comprometido con su función formadora y un agente dinamizador de experiencias educativas en las que prime el uso del lenguaje de manera funcional, dialógica, conversacional (Van Dijk, 1980). Un maestro que se acerca humanizado 
para compartir lecturas con el estudiante, para opinar y suscitar pareceres en sus discípulos a partir de las lecturas y reflexiones extraídas de poemas, novelas, ensayos, etc. (Dubois, 2011).

La educación exige un compromiso entre el docente con sus saberes, el contexto, sus estudiantes, sus mismos compañeros y sus estrategias de enseñanza (Girón Castro, Jiménez Camargo y Lizcano Rivera, 2005). Por otro lado, está la consideración de las prácticas de lectura crítica entendidas como parte de una experiencia transformadora en los maestros. Un maestro lector crítico, que sabe adentrarse en las profundidades del texto, que es capaz de desentrañar la esencia de las lecturas, es un maestro que suscita instancias de acercamiento a la lectura, a la escritura como prácticas mediadoras para la reflexión sobre la realidad, los fenómenos del mundo, la apreciación estética de lo que existe y la toma de decisiones fundadas en la formación y la transformación operada por la experiencia asumida desde la lectura crítica (Ferreiro, 1991; Ferreiro y Teberosky, 1981; Chevallard, 1998).

Partiendo de la revisión anterior, esta investigación se realizó con el propósito de auscultar la incidencia de la lectura crítica y el pensamiento reflexivo de los docentes de educación secundaria de una institución educativa de la ciudad de Cúcuta, en sus prácticas pedagógicas. Además tuvo en cuenta posturas desde las cuales se concibe no solo la lectura crítica y el pensamiento reflexivo, sino también las prácticas pedagógicas como objeto de estudio, logrando apoyarse en Zuluaga (1999), sus teorías y estudios, el sustento teórico necesario para orientar las acciones que permitieron definir un diseño de investigación ajustado a la naturaleza del objeto de estudio, con lo que, los aportes de Martínez Miguélez (2015), procuraron orientar la identificación de un paradigma y unas líneas desde las cuales desarrollar las acciones pertinentes para el desarrollo de las actividades de campo.

Tres objetivos esenciales se definieron para el desarrollo de la investigación: Identificar las características y posturas recurrentes de un docente lector en sus prácticas pedagógicas; clasificar las estrategias de animación y promoción de la lectura, importantes en la creación de ambientes de aprendizaje pertinentes; y determinar cuáles prácticas pedagógicas aportan al desarrollo del pensamiento crítico reflexivo.

El primer objetivo estuvo orientado a interpretar las prácticas pedagógicas de los docentes de básica secundaria, utilizando informantes claves de las áreas académicas de lengua castellana, ciencias sociales y religión, por ser docentes que mostraron inclinaciones por el hábito lector.

El segundo objetivo, a partir de la aplicación de las técnicas y los instrumentos de recolección de datos, consistió en identificar las diferentes estrategias utilizadas por los docentes para hacer de la lectura y la escritura, hábitos importantes en los estudiantes, que indujeran a pensar críticamente y actuar en consecuencia.

Con respecto al tercer objetivo, las prácticas pedagógicas que mejores resultados aportaron los docentes en la construcción de un pensamiento reflexivo fueron objeto de interpretación por las investigadoras en las diferentes fases del diseño de investigación.

\section{MÉTODO}

\subsection{PARADIGMA Y TIPO DE INVESTIGACIÓN}

El paradigma que orientó la investigación es el interpretativo: histórico-hermenéutico; y con el propósito de alcanzar el objetivo de la investigación, se empleó un enfoque 
cualitativo, ya que se pretendía llegar a caracterizar las prácticas de los docentes y este tipo de investigación se direcciona hacia la construcción de conocimiento acerca de la realidad social y cultural a partir de la descripción e interpretación de las perspectivas de los sujetos involucrados (Hurtado, 2010; Martínez Miguélez, 2009).

Este proceso dio pie para poder ahondar en las prácticas pedagógicas de los educadores a partir de sus relatos u observación de sus prácticas, teniendo en cuenta que una de las categorías identificadas en la investigación es la lectura crítica. El paradigma histórico hermenéutico se ha definido como el marco epistemológico que dio sentido a la investigación, desde la perspectiva de la naturaleza de los fenómenos y realidades que conforman el objeto de estudio, ya que están referidos a personas, a instituciones y a una realidad educativa que determina posturas y consideraciones especiales relacionadas con la educación, su calidad y la incidencia de las prácticas pedagógicas de los sujetos participantes que se involucraron en el proceso de investigación.

Con este tipo de paradigma se consiguió que la investigación desarrollara una teoría coherente que partiera de lo particular que ocurre en el salón de clase, a lo general; las hipótesis fueron generadas a partir del resultado del estudio, y fue de gran ayuda en esta investigación, ya que desde este paradigma se evaluó el desarrollo natural de los sucesos sin manipular la realidad, pues buscaba interpretar lo que se iba captando activamente, y por lo tanto la realidad se construyó a través de las interpretaciones de los participantes de la investigación (Hurtado, 2006).

\subsection{DISEÑO DE INVESTIGACIÓN CORRIENTE EPISTEMOLÓGICA: RACIONALISTA REALISTA Y UN DISEÑO HERMENÉUTICO}

El diseño hermenéutico se ajusta perfectamente con el objeto de investigación, pues se requirió hacer una observación minuciosa de las prácticas pedagógicas de los profesores de secundaria del colegio, procurando identificar en sus estilos de enseñanza y las metodologías utilizadas, esas estrategias de animación y promoción de la lectura y la escritura que podrían generar una lectura crítica en los estudiantes y promover el desarrollo del pensamiento crítico Martínez Miguélez (2009, 2015).

El uso de las técnicas de recolección de información diseñadas en la investigación, pretendían conseguir más elementos de estudio y análisis que posibilitaran interpretar mejor las prácticas pedagógicas de los docentes, así como sus hábitos personales relacionados con la lectura crítica y el pensamiento reflexivo. El diseño hermenéutico hizo posible un mayor acercamiento a esa realidad, de manera más efectiva, para entender sus posturas como maestros, el uso del lenguaje, el manejo de recursos, sus tics, sus gestos y hasta la manera de establecer contacto con los estudiantes en el aula (Martínez Miguélez, 2009, 2015). Se concibieron en esta investigación tres fases: fase de observación, fase de descripción y fase de análisis y contrastación (Martínez Miguélez, 2009).

\subsection{PARTICIPANTES}

La población de esta investigación fue una institución educativa privada conformada por 489 estudiantes y 32 profesores. La muestra fue seleccionada de forma intencional, seleccionando 4 docentes, bajo criterios de selección. Los criterios para la selección de los mismos consideraron: Ser docentes de básica secundaria y media de las áreas de lengua 
castellana, de ciencias sociales y de religión, y tener la disponibilidad para participar de dicha investigación (Martínez Miguélez, 2009). Para la sistematización de la información que se recopiló, se asignaron los siguientes códigos DC1, DC2, DC3 y DC4 para hacer referencia al Docente 1, Docente 2, Docente 3, y Docente 4, respectivamente.

\subsection{TÉCNICAS E INSTRUMENTOS DE RECOLECCIÓN DE INFORMACIÓN}

Bajo el diseño de investigación seleccionado y acorde con los objetivos de investigación planteados en el estudio desarrollado, se utilizaron las técnicas de observación no participante y la entrevista semiestructurada. En consonancia con lo planteado, los instrumentos utilizados en este proyecto fueron: Mapeo, rutas de observación, fichas de observación, diarios de campo, guion de entrevista semi-estructurada, que tienen el fin de constatar de manera objetiva el proceso de cada uno de profesores investigados (Martínez Miguélez, 2009, 2015).

\subsubsection{Observación no participante}

Esta técnica permitió, de manera directa, obtener información concreta relacionada con el primer objetivo específico de la investigación: Identificar las características y posturas recurrentes de un docente lector en sus prácticas pedagógicas, permitiendo a los investigadores reconocer in situ las rutinas pedagógicas de los docentes, sus actitudes en el aula, sus hábitos de enseñanza y las disposiciones para asumir los encuentros educativos con sus estudiantes, así como la forma como planean u organizan las secuencias didácticas de su disciplina académica (Martínez Miguélez, 2009, 2015).

Los pasos que se llevaron a cabo para realizar el proceso de observación fueron (Quintana y Montgomery, 2006):
1. Mapeo
2. Planeación de rutas de observación.
3. Fichas de rutas de observación.
4. Diarios de campo.

\subsubsection{La Entrevista semi-estructurada}

La entrevista semiestructurada, por su naturaleza y su fácil utilización dentro del enfoque cualitativo, fue de vital importancia en la investigación, ya que permitió confrontar visiones y perspectivas en los sujetos participantes, asumidos como profesores de área, con sus propios imaginarios como personas. De estas percepciones, fue posible extraer apreciaciones de singular valor cuando se analizaron los datos e informaciones aportadas por la observación de la práctica pedagógica concreta en el aula. La entrevista, permitió, asimismo, considerar aspectos importantes relacionados con la formación disciplinar de los profesores, así como de sus concepciones sobre la práctica pedagógica misma (Martínez Miguélez, 2009, 2015).

\subsection{TÉCNICA DE PROCESAMIENTO Y ANÁLISIS DE INFORMACIÓN}

Esta etapa de la investigación cualitativa se realizó luego de haber recogido y descrito un buen conjunto de material protocolar (primario), en entrevistas, observaciones, 
grabaciones y anotaciones, que se consideraron suficiente para emprender una sólida categorización o clasificación que, a su vez, pudo nutrir un buen análisis, interpretación y teorización y conducir a resultados valiosos.

Este proceso de análisis de la información se halla en la perspectiva de la creación o invención de una teoría interpretativa de la realidad o fenómeno estudiado durante cada una de las fases de la investigación. Gran parte de los elementos cohesivos del discurso que hubieron de configurarse en el análisis de la información emergieron del material recopilado en grabaciones, anotaciones, entrevistas, conversaciones, diarios de campo y demás (Martínez Miguélez, 2009, 2015).

La complejidad del proceso ha implicado partir de unos supuestos o categorías engarzadas dentro del contexto del objeto de la investigación y asociadas a los procesos lógicos relacionados con las prácticas pedagógicas, enfocadas desde la perspectiva de la lectura crítica como elemento detonante, para usar una expresión alegórica o metafórica, según Martínez Miguélez (2009, 2015).

\section{RESULTADOS}

\subsection{IDENTIFICAR LAS CARACTERÍSTICAS Y POSTURAS RECURRENTES DE UN DOCENTE} LECTOR EN SUS PRÁCTICAS PEDAGÓGICAS

En este primer apartado se presentan los resultados que dan cuenta de la apuesta establecida en el primer objetivo de la investigación: Identificar las características y posturas recurrentes de un docente lector en sus prácticas pedagógicas. Estas prácticas o experiencias educativas están relacionadas con el saber pedagógico al que hace Zuluaga (1999) quien menciona que "en el centro del proceso de enseñanza es necesario colocar al maestro y su saber para reconocer todos los elementos que componen este proceso, de por sí complejo, y para devolver a la enseñanza el estatuto de práctica de saber entre prácticas” (p. 14).

De esta manera, se hace una identificación de una serie de categorías o hallazgos teóricos recurrentes en las observaciones realizadas. Las categorías teóricas axiales que emergieron en un primer momento de análisis (Lectura y escritura, Procesos de conceptualización, Criticidad y análisis, Expresión oral, Enseñanza-aprendizaje, Ambiente escolar, Metodología, Evaluación, Estrategias didácticas), muestran su valor, ya que permitieron ordenar de forma sistemática tanto los procesos teóricos, como sus análisis y posterior interpretación, hecho que como se mostrará a continuación, permitió reconocer el nivel de influencia. 
Tabla 1. Códigos usados en matriz de categorización proceso de observación

\begin{tabular}{|c|c|c|}
\hline \multicolumn{3}{|c|}{ Códigos matriz proceso de observación } \\
\hline Dc & Diario de campo & Observación en el aula \\
\hline Ob1 & Observación 1 & Observación en el aula \\
\hline Ob2 & Observación 2 & Observación en otros ambientes del colegio. \\
\hline Ob3 & Observación 3 & Religión $4^{\circ}$ a $11^{\circ}$ \\
\hline D1 & Docente 1 & Lengua castellana y economía y política $7^{\circ}, 8^{\circ}, 9^{\circ}, 10^{\circ}, 11^{\circ}$ \\
\hline D2 & Docente 2 & Lengua castellana $6^{\circ}, 7^{\circ}, 8^{\circ}$ \\
\hline D3 & Docente 3 & \\
\hline D4 & Docente 4 & \\
\hline
\end{tabular}

Tabla 2. Códigos usados en matriz de categorización proceso de entrevistas

\begin{tabular}{|c|c|c|}
\hline \multicolumn{3}{|c|}{ Códigos matriz proceso de entrevistas } \\
\hline E & Entrevista & \\
\hline Ed1 & Entrevista docente 1 & Sociales y economía y política $7^{\circ}, 8^{\circ}, 9^{\circ}, 10^{\circ}, 11^{\circ}$ \\
\hline Ed2 & Entrevista docente 2 & Religión $4^{\circ}$ a $11^{\circ}$ \\
\hline Ed3 & Entrevista docente 3 & Lengua castellana $9^{\circ}, 10^{\circ}, 11^{\circ}$ \\
\hline Ed4 & Entrevista docente 4 & Lengua castellana $6^{\circ}, 7^{\circ}, 8^{\circ}$ \\
\hline
\end{tabular}

\subsubsection{Categorías inductivas que emergieron del análisis de la información}

La categoría básica de la Investigación identificada como Lectura Crítica, ha permitido orientar el desarrollo de un proceso de reconocimiento de la información recabada por medio de los instrumentos de recolección diseñados y aplicados con los participantes en esta experiencia, propiciando la reflexión sobre los datos suministrados por los informantes claves, de la que emergieron categorías inductivas como: Procesos de conceptualización y Lectura y escritura.

Estas dos categorías conceptuales posibilitan establecer conexiones lógicas dentro del campo de la Lectura Crítica como categoría básica de la investigación, orientada al desarrollo del primer objetivo de la investigación: Identificar las características y posturas recurrentes de un docente lector en sus prácticas pedagógicas, entendiendo, que de acuerdo con la información suministrada en estos instrumentos como el diario de campo y las rutas de observación, correspondientes con las técnicas de Observación no participante, estas categorías emergen como elementos comunes del discurso de los docentes observados, que evidencian una recurrencia a estas categorías que merecen ser explicadas en contexto. 
Tabla 3. Cuadro de recurrencias en categorías de observación

\begin{tabular}{|c|c|c|c|c|}
\hline $\begin{array}{l}\text { CATEGORÍAS } \\
\text { BÁSICAS }\end{array}$ & $\begin{array}{l}\text { CATEGORÍAS } \\
\text { AXIALES }\end{array}$ & $\begin{array}{l}\text { CATEGORÍAS } \\
\text { INDUCTIVAS }\end{array}$ & $\begin{array}{c}\mathrm{N}^{\circ} \text { DE } \\
\text { RECURRENCIAS }\end{array}$ & CÓDIGO \\
\hline \multirow{3}{*}{$\begin{array}{l}\text { LECTURA CRÍTICA } \\
\text { (6) }\end{array}$} & \multirow{2}{*}{$\begin{array}{c}\text { Lectura y escritura } \\
\text { (3) }\end{array}$} & Literatura & 1 & DCD2-OB2-58 \\
\hline & & $\begin{array}{c}\text { Comprensión } \\
\text { lectora }\end{array}$ & 2 & $\begin{array}{l}\text { DCD2-OB2-55 } \\
\text { DCD3-OB1-69 }\end{array}$ \\
\hline & $\begin{array}{c}\text { Procesos de } \\
\text { conceptualización } \\
\text { (3) }\end{array}$ & $\begin{array}{l}\text { Recomendaciones } \\
\text { pedagógicas }\end{array}$ & 3 & $\begin{array}{l}\text { DCD1-OB3-25 } \\
\text { DCD1-OB3-29 } \\
\text { DCD3-OB3-87 }\end{array}$ \\
\hline \multirow{7}{*}{$\begin{array}{l}\text { PENSAMIENTO } \\
\text { REFLEXIVO (16) }\end{array}$} & \multirow{4}{*}{$\begin{array}{l}\text { Criticidad y } \\
\text { análisis(9) }\end{array}$} & $\begin{array}{l}\text { Orientación del } \\
\text { trabajo }\end{array}$ & 1 & DCD2-OB1-52 \\
\hline & & $\begin{array}{c}\text { Recomendaciones } \\
\text { pedagógicas }\end{array}$ & 3 & $\begin{array}{l}\text { DCD1-OB3-26 } \\
\text { DCD3-OB3-83 } \\
\text { DCD3-OB3-85 }\end{array}$ \\
\hline & & Discusión & 2 & $\begin{array}{l}\text { DCD1-OB3-30 } \\
\text { DCD2-OB3-62 }\end{array}$ \\
\hline & & $\begin{array}{l}\text { Orientación del } \\
\text { aprendizaje }\end{array}$ & 3 & $\begin{array}{l}\text { DCD3-OB3-88 } \\
\text { DCD3-OB3-89 } \\
\text { DCD3-OB3-90 }\end{array}$ \\
\hline & \multirow[b]{2}{*}{ Expresión oral (5) } & Discusión & 2 & $\begin{array}{l}\text { DCD1-OB3-27 } \\
\text { DCD2-OB3-60 }\end{array}$ \\
\hline & & $\begin{array}{l}\text { Orientación del } \\
\text { aprendizaje }\end{array}$ & 3 & $\begin{array}{r}\text { DCD3-OB1-70 } \\
\text { DCD3-OB3-80 } \\
\text { DCD4-OB3-112 }\end{array}$ \\
\hline & $\begin{array}{l}\text { Enseñanza- } \\
\text { aprendizaje(2) }\end{array}$ & $\begin{array}{l}\text { Organización del } \\
\text { pensamiento }\end{array}$ & 2 & $\begin{array}{c}\text { DCD1-OB3-2 } \\
\text { DCD1-OB3-24 }\end{array}$ \\
\hline
\end{tabular}




\begin{tabular}{|c|c|c|c|c|}
\hline & & $\begin{array}{l}\text { Orientación del } \\
\text { trabajo }\end{array}$ & 2 & $\begin{array}{l}\text { DCD2-OB1-47 } \\
\text { DCD3-OB3-81 }\end{array}$ \\
\hline & $\begin{array}{c}\text { Ambiente escolar } \\
\text { (18) }\end{array}$ & $\begin{array}{l}\text { Organización del } \\
\text { grupo }\end{array}$ & 11 & $\begin{array}{c}\text { DCD1-OB1-1 } \\
\text { DCD1-OB4-31 } \\
\text { DCD2-OB1-40 } \\
\text { DCD2-OB1-44 } \\
\text { DCD2-OB1-48 } \\
\text { DCD2-OB2-53 } \\
\text { DCD2-OB3-61 } \\
\text { DCD3-OB2-74 } \\
\text { DCD4-OB1-91 } \\
\text { DCD4-OB2-98 } \\
\text { DCD4-OB2-99 }\end{array}$ \\
\hline & & $\begin{array}{c}\text { Recomendaciones } \\
\text { pedagógicas }\end{array}$ & 1 & DCD1-OB4-35 \\
\hline & & Plan lector & 3 & $\begin{array}{l}\text { DCD4-OB2-100 } \\
\text { DCD4-OB2-101 } \\
\text { DCD4-OB2-102 }\end{array}$ \\
\hline & & Expresión cultural & 1 & DCD4-OB3-103 \\
\hline & & Discusión & 1 & DCD2-OB1-51 \\
\hline $\begin{array}{c}\text { PRÁCTICAS } \\
\text { PEDAGÓGICAS(90) }\end{array}$ & Metodología(23 & $\begin{array}{l}\text { Orientación del } \\
\text { trabajo }\end{array}$ & 13 & $\begin{array}{c}\text { DCD1-OB1-2 } \\
\text { DCD1-OB1-4 } \\
\text { DCD2-OB1-43 } \\
\text { DCD2-OB1-45 } \\
\text { DCD2-OB1-46 } \\
\text { DCD2-OB2-54 } \\
\text { DCD2-OB2-58 } \\
\text { DCD2-OB2-59 } \\
\text { DCD2-OB3-63 } \\
\text { DCD2-OB3-64 } \\
\text { DCD2-OB3-65 } \\
\text { DCD2-OB3-66 } \\
\text { DCD3-OB1-67 }\end{array}$ \\
\hline & & $\begin{array}{l}\text { Orientaciones } \\
\text { metodológicas }\end{array}$ & 1 & DCD1-OB1-3 \\
\hline & & $\begin{array}{c}\text { Recomendaciones } \\
\text { pedagógicas }\end{array}$ & 7 & $\begin{array}{c}\text { DCD1-OB1-9 } \\
\text { DCD1-OB2-19 } \\
\text { DCD1-OB2-20 } \\
\text { DCD2-OB1-49 } \\
\text { DCD2-OB1-50 } \\
\text { DCD4-OB1-92 } \\
\text { DCD4-OB3-111 }\end{array}$ \\
\hline & & Planeación escolar & 1 & DCD3-OB3-86 \\
\hline
\end{tabular}




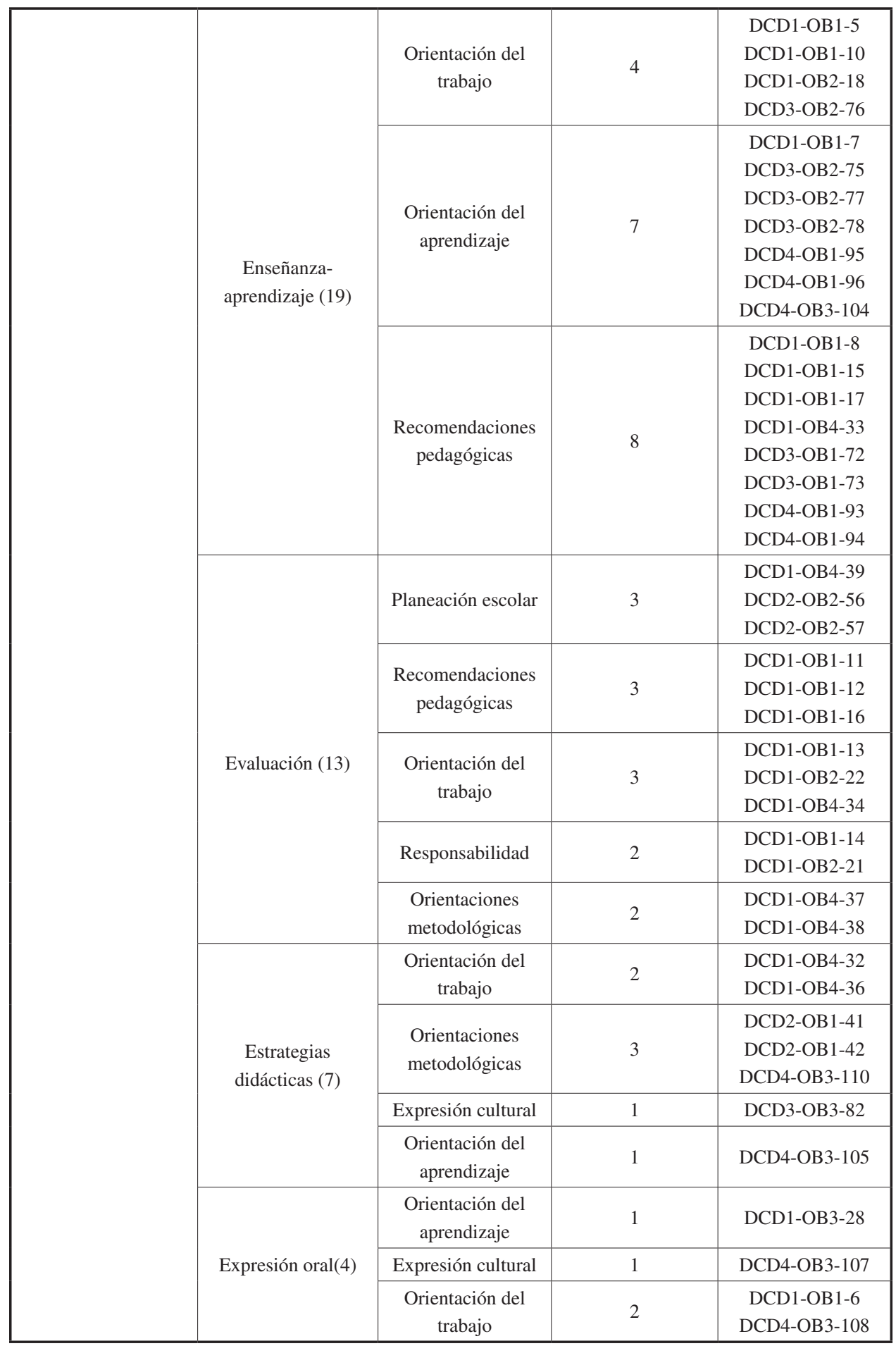




\begin{tabular}{|l|c|c|c|c|}
\hline \multirow{7}{*}{$\begin{array}{c}\text { Lectura y } \\
\text { escritura(5) }\end{array}$} & $\begin{array}{c}\text { Expresión cultural } \\
\text { Recomendaciones } \\
\text { pedagógicas }\end{array}$ & Plan lector & 1 & DCD3-OB3-79 \\
\cline { 3 - 5 } & & Responsabilidad & 1 & $\begin{array}{c}\text { DCD3-OB3-84 } \\
\text { DCD4-OB3-109 }\end{array}$ \\
\cline { 2 - 5 } & $\begin{array}{c}\text { Procesos de } \\
\text { conceptualización(1) }\end{array}$ & $\begin{array}{c}\text { Recomendaciones } \\
\text { pedagógicas }\end{array}$ & 1 & DCD4-OB1-97 \\
\hline & & 1 & DCD4-OB3-106 \\
\hline
\end{tabular}

\subsection{CLASIFICAR LAS ESTRATEGIAS DE ANIMACIÓN Y PROMOCIÓN DE LA LECTURA}

El segundo objetivo de la investigación, clasificar las estrategias de animación y promoción de la lectura, importantes en la creación de ambientes de aprendizajes pertinentes, está relacionado con la observación e interpretación de las apuestas didácticas que los maestros utilizan en el aula de clase para hacer la lectura una herramienta importante en la orientación de procesos para propiciar aprendizajes efectivos en los estudiantes. En el desarrollo de esta apuesta investigativa se hizo uso de la técnica de investigación entrevista semiestructurada, que partiendo de la aplicación del instrumento guion de entrevista, permitió indagar las percepciones, posturas y apreciaciones de los informantes claves de la investigación, correspondientes a los docentes de Ciencias sociales y Filosofía (D1), docente de Religión (D2), docente de Lengua castellana (D3) y docente de Lengua castellana (D4), nivel de secundaria y media, sobre las estrategias de animación y promoción de la lectura implementadas en sus prácticas pedagógicas. 
Tabla 4. Cuadro de recurrencias en categorías de entrevistas

\begin{tabular}{|c|c|c|c|c|}
\hline $\begin{array}{l}\text { CATEGORÍAS } \\
\text { BÁSICAS }\end{array}$ & $\begin{array}{l}\text { CATEGORÍAS } \\
\text { AXIALES }\end{array}$ & $\begin{array}{l}\text { CATEGORÍAS } \\
\text { INDUCTIVAS }\end{array}$ & $\begin{array}{c}\mathrm{N}^{\circ} \\
\text { RECURRENCIAS }\end{array}$ & CÓDIGO \\
\hline \multirow{16}{*}{$\begin{array}{l}\text { LECTURA } \\
\text { CRÍTICA (22) }\end{array}$} & $\begin{array}{l}\text { Actualización } \\
\text { pedagógica (2) }\end{array}$ & $\begin{array}{l}\text { Motivación a } \\
\text { aprender (2) }\end{array}$ & 2 & $\begin{array}{l}\text { ED3-47 } \\
\text { ED4-70 }\end{array}$ \\
\hline & $\begin{array}{c}\text { Animación a la } \\
\text { lectura (1) }\end{array}$ & $\begin{array}{l}\text { Comprensión } \\
\text { lectora(1) }\end{array}$ & 1 & ED2-28 \\
\hline & \multirow{2}{*}{$\begin{array}{c}\text { Enseñanza } \\
\text { aprendizaje (3) }\end{array}$} & $\begin{array}{c}\text { Saber } \\
\text { pedagógico(1) }\end{array}$ & 1 & ED3-43 \\
\hline & & $\begin{array}{l}\text { Orientación al } \\
\text { aprendizaje(2) }\end{array}$ & 1 & $\begin{array}{l}\text { ED3-45 } \\
\text { ED3-46 }\end{array}$ \\
\hline & \multirow{3}{*}{$\begin{array}{l}\text { Formación lectora } \\
\text { (3) }\end{array}$} & $\begin{array}{c}\text { Comprensión } \\
\text { lectora (1) }\end{array}$ & 1 & ED1-1 \\
\hline & & Proceso lector(1) & 1 & ED1-7 \\
\hline & & $\begin{array}{l}\text { Ambientes de } \\
\text { lectura(1) }\end{array}$ & 1 & ED2-24 \\
\hline & \multirow[b]{3}{*}{ Hábito lector (4) } & $\begin{array}{l}\text { Desinterés por la } \\
\text { lectura(1) }\end{array}$ & 1 & ED1-5 \\
\hline & & Proceso lector(1) & 1 & ED1-6 \\
\hline & & $\begin{array}{l}\text { Motivación a } \\
\text { aprender (2) }\end{array}$ & 2 & $\begin{array}{l}\text { ED2-26 } \\
\text { ED3-44 }\end{array}$ \\
\hline & $\begin{array}{c}\text { Proceso de } \\
\text { conceptualización } \\
\text { (3) }\end{array}$ & $\begin{array}{l}\text { Imprecisión } \\
\text { conceptual (3) }\end{array}$ & 3 & $\begin{array}{l}\text { ED2-27 } \\
\text { ED3-48 } \\
\text { ED4-71 }\end{array}$ \\
\hline & \multirow[b]{4}{*}{$\begin{array}{c}\text { Promoción de la } \\
\text { lectura(4) }\end{array}$} & Sugerir autores (1) & 1 & ED1-2 \\
\hline & & Indagación (1) & 1 & ED1-3 \\
\hline & & Curiosidad (1) & 1 & ED1-4 \\
\hline & & $\begin{array}{c}\text { Comprensión } \\
\text { lectora(1) }\end{array}$ & 1 & ED4-69 \\
\hline & $\begin{array}{c}\text { Promoción lectora } \\
\text { (2) }\end{array}$ & $\begin{array}{l}\text { Comprensión } \\
\text { lectora (2) }\end{array}$ & 2 & $\begin{array}{l}\text { ED2-23 } \\
\text { ED2-25 }\end{array}$ \\
\hline
\end{tabular}


LECTURA CRÍTICA Y PENSAMIENTO REFLEXIVO EN LA PRÁCTICA PEDAGÓGICA DE PROFESORES DE BÁSICA SECUNDARIA

\begin{tabular}{|c|c|c|c|c|}
\hline \multirow{19}{*}{$\begin{array}{l}\text { PENSAMIENTO } \\
\text { REFLEXIVO (23) }\end{array}$} & $\begin{array}{c}\text { Animación a la } \\
\text { lectura (1) }\end{array}$ & $\begin{array}{c}\text { Activar } \\
\text { imaginación(1) }\end{array}$ & 1 & ED2-32 \\
\hline & \multirow{13}{*}{$\begin{array}{l}\text { Criticidad y análisis } \\
\qquad(15)\end{array}$} & Discusión (2) & 2 & $\begin{array}{l}\text { ED4-72 } \\
\text { ED4-73 }\end{array}$ \\
\hline & & $\begin{array}{c}\text { Ejemplo y } \\
\text { motivación (1) }\end{array}$ & 1 & ED2-31 \\
\hline & & $\begin{array}{l}\text { Formación } \\
\text { personal (1) }\end{array}$ & 1 & ED1-12 \\
\hline & & Indagación (1) & 1 & ED1-11 \\
\hline & & Inferencia (1) & 1 & ED2-29 \\
\hline & & $\begin{array}{c}\text { Inferir y } \\
\text { argumentar (1) }\end{array}$ & 1 & ED1-8 \\
\hline & & $\begin{array}{l}\text { Orientación al } \\
\text { aprendizaje (1) }\end{array}$ & 1 & ED2-30 \\
\hline & & $\begin{array}{l}\text { Problematización } \\
\text { (1) }\end{array}$ & 1 & ED1-9 \\
\hline & & $\begin{array}{c}\text { Recomendaciones } \\
\text { pedagógicas(2) }\end{array}$ & 2 & $\begin{array}{l}\text { ED3-51 } \\
\text { ED3-52 }\end{array}$ \\
\hline & & $\begin{array}{l}\text { Redacción textual } \\
\text { (1) }\end{array}$ & 1 & ED3-49 \\
\hline & & $\begin{array}{l}\text { Reflexión de la } \\
\text { escritura (1) }\end{array}$ & 1 & ED3-55 \\
\hline & & $\begin{array}{l}\text { Selección de textos } \\
\text { (1) }\end{array}$ & 1 & ED1-10 \\
\hline & & $\begin{array}{l}\text { Vivenciar la } \\
\text { realidad (1) }\end{array}$ & 1 & ED4-75 \\
\hline & $\begin{array}{c}\text { Enseñanza } \\
\text { aprendizaje (1) }\end{array}$ & $\begin{array}{l}\text { Organización del } \\
\text { pensamiento (1) }\end{array}$ & 1 & ED4-74 \\
\hline & \multirow[t]{2}{*}{ Expresión oral(4) } & $\begin{array}{l}\text { Diálogo de } \\
\text { saberes(3) }\end{array}$ & 3 & $\begin{array}{l}\text { ED3-53 } \\
\text { ED3-54 } \\
\text { ED4-76 }\end{array}$ \\
\hline & & $\begin{array}{l}\text { Diálogo de } \\
\text { saberes(1) }\end{array}$ & 1 & ED2-33 \\
\hline & $\begin{array}{c}\text { Expresión oral y } \\
\text { escrita(1) }\end{array}$ & $\begin{array}{c}\text { Dialogo y } \\
\text { confrontación de } \\
\text { saberes (1) }\end{array}$ & 1 & ED2-34 \\
\hline & $\begin{array}{l}\text { Organización del } \\
\text { pensamiento (1) }\end{array}$ & $\begin{array}{c}\text { Mediación de la } \\
\text { lectura(1) }\end{array}$ & 1 & ED3-50 \\
\hline
\end{tabular}




\begin{tabular}{|c|c|c|c|c|}
\hline \multirow{26}{*}{$\begin{array}{c}\text { PRÁCTICAS } \\
\text { PEDAGÓGICAS } \\
(36)\end{array}$} & \multirow{4}{*}{ Ambiente escolar(5) } & $\begin{array}{l}\text { Motivación a } \\
\text { aprender (1) }\end{array}$ & 1 & ED3-42 \\
\hline & & $\begin{array}{l}\text { Orientación al } \\
\text { trabajo (1) }\end{array}$ & 1 & ED4-80 \\
\hline & & Participación (1) & 1 & ED2-37 \\
\hline & & Plan lector (2) & 2 & $\begin{array}{l}\text { ED3-65 } \\
\text { ED4-66 }\end{array}$ \\
\hline & \multirow{11}{*}{$\begin{array}{c}\text { Enseñanza } \\
\text { aprendizaje(14) }\end{array}$} & $\begin{array}{l}\text { Calidad educativa } \\
\text { (1) }\end{array}$ & 1 & ED1-20 \\
\hline & & $\begin{array}{c}\text { Diálogo y } \\
\text { discusión (1) }\end{array}$ & 1 & ED1-13 \\
\hline & & $\begin{array}{c}\text { Evaluación externa } \\
\text { (1) }\end{array}$ & 1 & ED1-19 \\
\hline & & $\begin{array}{c}\text { Exigencia } \\
\text { institucional (1) }\end{array}$ & 1 & ED2-39 \\
\hline & & $\begin{array}{l}\text { Formación } \\
\text { ciudadana (1) }\end{array}$ & 1 & ED2-18 \\
\hline & & $\begin{array}{l}\text { Lectura crítica y } \\
\text { creatividad (1) }\end{array}$ & 1 & ED2-41 \\
\hline & & $\begin{array}{l}\text { Orientación del } \\
\text { trabajo (3) }\end{array}$ & 3 & $\begin{array}{l}\text { ED2-40 } \\
\text { ED4-67 } \\
\text { ED4-77 }\end{array}$ \\
\hline & & $\begin{array}{l}\text { Orientaciones } \\
\text { metodológicas (1) }\end{array}$ & 1 & ED4-81 \\
\hline & & $\begin{array}{c}\text { Planeación escolar } \\
\text { (2) }\end{array}$ & 2 & $\begin{array}{l}\text { ED1-14 } \\
\text { ED3-60 }\end{array}$ \\
\hline & & $\begin{array}{l}\text { Recomendaciones } \\
\text { pedagógicas (1) }\end{array}$ & 1 & ED4-68 \\
\hline & & $\begin{array}{l}\text { Responsabilidad } \\
\text { (1) }\end{array}$ & 1 & ED3-63 \\
\hline & \multirow{4}{*}{$\begin{array}{c}\text { Estrategias } \\
\text { didácticas(8) }\end{array}$} & $\begin{array}{l}\text { Orientaciones } \\
\text { metodológicas(3) }\end{array}$ & 3 & $\begin{array}{l}\text { ED1-15 } \\
\text { ED3-57 } \\
\text { ED4-78 }\end{array}$ \\
\hline & & $\begin{array}{l}\text { Lectura y estudio } \\
\text { disciplinar ( } 3 \text { ) }\end{array}$ & 3 & $\begin{array}{l}\text { ED2-38 } \\
\text { ED3-61 } \\
\text { ED4-79 }\end{array}$ \\
\hline & & $\begin{array}{c}\text { Fortalecer procesos } \\
\text { de lectura (1) }\end{array}$ & 1 & ED1-17 \\
\hline & & Expresión oral (1) & 1 & ED3-58 \\
\hline & Evaluación (1) & $\begin{array}{l}\text { Orientación al } \\
\text { trabajo(1) }\end{array}$ & 1 & ED3-62 \\
\hline & $\begin{array}{c}\text { Formación } \\
\text { lectora(2) }\end{array}$ & $\begin{array}{c}\text { Orientación } \\
\text { metodológica(2) }\end{array}$ & 2 & $\begin{array}{l}\text { ED2-21 } \\
\text { ED2-22 }\end{array}$ \\
\hline & $\begin{array}{l}\text { Formación } \\
\text { personal(1) }\end{array}$ & $\begin{array}{c}\text { Literatura } \\
\text { contemporánea(1) }\end{array}$ & 1 & ED3-64 \\
\hline & \multirow{3}{*}{ Metodología (4) } & $\begin{array}{l}\text { Orientación del } \\
\text { trabajo (1) }\end{array}$ & 1 & ED2-35 \\
\hline & & $\begin{array}{l}\text { Orientaciones } \\
\text { metodológicas (1) }\end{array}$ & 1 & ED2-36 \\
\hline & & $\begin{array}{c}\text { Planeación escolar } \\
\text { (2) }\end{array}$ & 2 & $\begin{array}{l}\text { ED3-56 } \\
\text { ED3-59 }\end{array}$ \\
\hline & $\begin{array}{l}\text { Saber pedagógico } \\
\text { (1) }\end{array}$ & $\begin{array}{l}\text { Literatura y estudio } \\
\text { disciplinar (1) }\end{array}$ & 1 & ED1-16 \\
\hline
\end{tabular}



SECUNDARIA

Para hacer una efectiva ubicación contextual de este análisis es pertinente hacer un acercamiento conceptual a la lectura y los elementos con los cuales se hizo seguimiento a los procesos relacionados con los resultados arrojados por el instrumento de recolección de información que se ha implementado. Para esto se conceptualiza la lectura, entendida como un proceso global y complejo que va más allá de la simple decodificación mecánica de unos signos gráficos. No sólo se trata de identificar y nombrar correctamente palabras y frases, sino que, además, la lectura implica interpretar un texto, atribuirle un significado, comprenderlo. Y éste es un proceso dinámico en el que tiene lugar una interacción permanente entre lector, texto y contexto. El lector, para construir el significado utiliza la información que le proporciona el texto, sus conocimientos previos sobre el tema y realiza además una serie de operaciones mentales. Pero también en la construcción del significado que el sujeto lleva a cabo, intervienen una serie de factores afectivos y emocionales, vinculados a sus experiencias personales con la lectura (expectativas, necesidad satisfecha, identificación, placer proporcionado...) y a los estímulos y valores del medio social (presencia de modelos lectores, valoración social de la lectura, etc.).

Todos estos aspectos y factores que intervienen en la lectura fueron tenidos en cuenta a la hora de analizar las intervenciones de animación y promoción de la lectura. La Animación de la lectura, por su parte, constituye una categoría emergente de gran valor a la hora de hacer consideraciones especiales de análisis sobre los resultados obtenidos en este trabajo y concretamente en el desarrollo de la entrevista como técnica de investigación.

En este caso se refiere a la dimensión más personal e individual de la lectura. Se trataría de conectar lector y texto, de que se produzca una interacción entre ambos. En segundo lugar, en la segunda proposición de esta definición, se busca crear el gusto por la lectura. Aquí se matiza el carácter de ese vínculo, que vendrá determinado por el disfrute y la satisfacción personal del individuo o grupo. En tercer lugar, no conviene obviar la idea de que se pretende que el lector conecte con un material específico de lectura. Este es un aspecto que no habría que perder de vista en la animación y que con frecuencia es obviado en el desarrollo de multitud de actividades que tienen lugar en escuelas, bibliotecas y otros ámbitos. Talleres, representaciones teatrales, exposiciones, y otras, en las que el libro es el gran ausente, se presentan como propuestas válidas para crear vínculos entre lectores y textos.

Con respecto a la promoción de la lectura, el autor Yepes (2001) la define como esa "acción o conjunto de acciones dirigidas a acercar a un individuo o grupo a la lectura, elevándola a un nivel superior de uso, de tal modo que sea asumida como una herramienta indispensable en el ejercicio pleno de la condición vital y civil" (p. 187). Desde esta perspectiva, la promoción de la lectura se convierte en un proceso intencional y sistemático que abarca todo un conjunto de acciones que van desde el diseño de una política determinada, nacional, institucional, hasta el diseño de planes de acción concretos que incluyen entre otras opciones, las actividades de animación a la lectura.

La promoción se entendería como una idea más genérica que pretendería articular relaciones de continuidad y coherencia entre distintas acciones. Se trataría de vincular necesidades sociales, objetivos, planes, recursos y resultados en el intento no sólo de acercar a unos materiales determinados sino de hacer de la lectura una práctica habitual, cotidiana y accesible (física, afectiva e intelectualmente) para cualquier ciudadano y para ello es preciso llevar a cabo otro tipo de acciones además de las actividades o planes de animación. 
Como se ha visto, el comportamiento lector o no lector de las personas está determinado e influido por muchos factores, y en el camino de la promoción habrá que hacer frente a todos los elementos disuasorios que conducen a las personas a adoptar una actitud pasiva ante la lectura. Transformar la imagen social, cultural, y en el contexto educativo, que existe de la lectura, y por ese motivo intentar aislar los conflictos en relación con la lectura, paliar las carencias y desigualdades en el acceso, establecer sus causas y consecuencias y revisar los procedimientos que han posibilitado su inserción en el mundo escolar de manera funcional y efectiva, estuvieron presentes en el análisis de estos resultados.

Estas consideraciones conceptuales posibilitan hacer una mirada concreta a la realidad educativa identificada en las prácticas pedagógicas de los docentes entrevistados, quienes, en primera instancia asumen la lectura como instrumento, como herramienta de trabajo de vital valor en la construcción de saberes. Desde esta perspectiva, la lectura entonces es un vehículo privilegiado que la institución anima y promueve desde varias instancias, en las que los docentes son agentes dinámicos importantes en el diseño de actividades y procesos que instrumentalizan la lectura, en todos sus niveles, para la creación de ambientes de trabajo funcionales para la formación de la criticidad, el análisis y el pensamiento reflexivo.

\subsection{DETERMINAR CUÁLES PRÁCTICAS PEDAGÓGICAS APORTABAN AL DESARROLLO DEL PENSAMIENTO CRÍTICO REFLEXIVO}

Como puede apreciarse, la investigación, al retomar las observaciones y la información de los procesos desarrollados con respecto a los dos primeros objetivos específicos planteados, se estaba dando cumplimiento a la intencionalidad de determinar cuáles prácticas pedagógicas aportaban al desarrollo del pensamiento crítico reflexivo. Y esas prácticas pedagógicas concretas están asociadas con esas categorías axiales identificadas esencialmente en los ejes categoriales selectivos de Pensamiento reflexivo y Práctica pedagógica, reconocidas, de manera integral en la utilización de las dos técnicas de investigación, como: Criticidad y análisis, Expresión oral, Enseñanza-aprendizaje, Formación lectora, Metodología, Estrategias didácticas, Ambiente escolar. No se registran algunas categorías emergentes cuya frecuencia fue limitada a una sola aparición en el análisis.

Aparte de que no se concretan las estrategias didácticas solicitadas en la pregunta de la entrevista, sí pone de manifiesto la importancia del desarrollo de estrategias didácticas innovadoras, esenciales dentro de la práctica pedagógica para el fortalecimiento de saberes, apropiación de aprendizajes, indispensables en la formación del pensamiento reflexivo, como fin ulterior de los procesos educativos que se ejecutan dentro de la cotidianidad escolar.

El maestro es lo que hace en el aula. La actividad profesional del maestro se hace realidad en su práctica pedagógica. Ella se configura en el conjunto de conocimientos que dan forma a los contenidos que se enseñan, pero también en la formación de habilidades necesarias para enfrentar el hecho pedagógico. La naturaleza de esos conocimientos y habilidades da autonomía al docente y define las acciones de su situación profesional. El maestro es ese sujeto autónomo dotado de habilidades específicas y conocimientos racionales o explícitos que se ponen en práctica al momento de mediar en una situación de enseñanza-aprendizaje.

Es la realidad vivenciada en la institución educativa, en donde los informantes claves de la investigación (D1: docente de Sociales y Filosofía, D2: docente de Religión, D3: 
docente de lengua castellana, y D4: docente de lengua castellana), gracias a la Observación no participante y la entrevista semiestructurada, utilizadas como técnicas de investigación cualitativa, permitieron reconocer la importancia de la formación del pensamiento reflexivo en los estudiantes, a partir de la implementación de estrategias didácticas y metodologías activas participativas, mediadas también por el diálogo, la indagación y la discusión en el aula, desde la comprensión de las prácticas pedagógicas de los maestros que asumen la lectura como vehículo educativo privilegiado, con mayor apropiación desde la experiencia del docente D1, como lector crítico.

En concreto, la práctica pedagógica es ese hacer que hace del maestro lo que es, asumiendo que el acto de enseñar es también un acto de aprendizaje, que se construye cada día en el aula. La institución educativa destaca como espacio institucional y establece los nexos necesarios instrumentales para propiciar el clima idóneo para que la lectura crítica, el pensamiento reflexivo y la práctica pedagógica constituyan una sola realidad para repensar el diálogo de enseñar y aprender. La práctica pedagógica, como plantea Zuluaga (1996) "permite describir e historizar el proceso de institucionalización del saber pedagógico, en el que interactúan las instituciones, los sujetos, los discursos y las tecnologías de poder que en cada formación social han dado materialidad a la función de enseñar" (p. 125).

Las prácticas pedagógicas identificadas en la experiencia de observación y en las entrevistas realizadas con informantes claves D1, D2, D3 y D4, dentro de esta investigación, se clasifican de acuerdo con la funcionalidad en el desarrollo de competencias.

Estas prácticas pedagógicas son situadas, en el sentido de que los docentes procuran siempre auscultar la realidad a partir de las percepciones de los estudiantes, reconocidas en sus encuentros conversacionales. Constituyen prácticas de indagación orientadas a la búsqueda de la verdad, no de lo real, sino de la realidad.

Las prácticas pedagógicas son humanísticas, ya que exploran las verdades de cada uno, asumidos como seres humanos, como personas que buscan identificarse con ellos mismos a través de los instrumentos y medios que la escolaridad ofrece, en donde el maestro funge de mediador, a partir de la pregunta, de la indagación sistemática.

Todas estas prácticas se concretan en la utilización efectiva de estrategias didácticas con un alto sentido pedagógico, representadas en procesos de lectura y de formación del pensamiento reflexivo.

En procesos de formación lectora como propósito institucional y como vehículo educativo privilegiado para el estímulo del pensamiento de la imaginación y herramienta pedagógica en la apropiación del lenguaje, útil para la argumentación en razón de la experiencia de vida que representa la literatura y otros textos, se hallan estrategias con sello institucional.

En Animación de lectura: comentarios de textos, de libros, de autores; discusión sobre películas, sus temas, argumentos, escenas; invitación a foros, debates y charlas.

En Promoción de lectura: mesas redondas sobre un autor; lecturas de fragmentos de obras literarias; lecturas comentadas sobre textos de filosofía; debates sobre temas de la vida política del país, reflejados en diarios o revistas; producción de textos a manera de comentarios sobre autores o libros leídos; análisis literarios de obras sugeridas.

En procesos de formación crítica y estructuración del pensamiento, las prácticas pedagógicas estuvieron orientadas, dentro de las experiencias observadas, a fortalecer la autonomía y la criticidad, a través de la expresión oral, mediante estrategias como: el diálogo docente-estudiante; privilegio en el encuentro dialógico del proceso conversacional; 
utilización de la indagación como detonante de ideas y posturas innovadoras en los interlocutores; promoción de la expresión oral y el discurso, mediante la exploración de la oratoria, los juegos teatrales y la cuentería.

\section{DISCUSIÓN Y CONCLUSIONES}

El análisis e interpretación de los datos se apoyó en tres categorías selectivas enmarcadas en los referentes que constituyen la fundamentación teórica de la investigación: Lectura crítica, Pensamiento reflexivo y Práctica pedagógica. En estas tres categorías, se han identificado categorías emergentes como la Formación lectora, Promoción de la lectura, Hábito lector, Proceso de conceptualización, Enseñanza-aprendizaje y Actualización pedagógica (Cassany, 2016; Zuluaga, 1996), para hacer alusión a la primera categoría selectiva, asociada directamente con el desarrollo del primer objetivo de investigación, identificar las características y posturas recurrentes de un docente lector en sus prácticas pedagógicas, en la que se precisan los siguientes hallazgos:

La lectura crítica no constituye una práctica efectiva en todos los docentes lectores investigados. Solo uno de ellos demuestra poseer un pensamiento reflexivo avanzado, facultad que manifiesta en su discurso, uso del lenguaje y posturas críticas con respecto a la realidad educativa, social y política. Recurre generalmente al texto, a los autores para suscitar la participación de sus estudiantes en las sesiones de clase, utilizando la indagación y la discusión en el aula, por lo que sus prácticas pedagógicas son relevantes, interesantes, motivadoras para los estudiantes (Campos, 2007; Cassany, 2013; Parodi, 2003).

Los docentes que poseen hábito lector, a pesar de que sus lecturas no superan el nivel de lectura por placer, lectura instrumental e informativa, asumen con propiedad su saber pedagógico para orientar procesos educativos ajustados a los tiempos y secuencias institucionales, utilizando con alguna regularidad el diálogo con enfoque conversacional en sus encuentros, para promover instancias enseñanza-aprendizaje (Munita, 2016; Tovar, 2009).

El segundo objetivo de la investigación buscaba clasificar las estrategias de animación y promoción de la lectura, importantes en la creación de ambientes de aprendizaje pertinentes, constituye un componente muy importante del proceso, asociado con el eje categorial Pensamiento reflexivo (Boisvert, 2004). Se agrupan en torno de esta categoría selectiva, categorías emergentes como Criticidad y análisis, Expresión oral, Enseñanzaaprendizaje (Ferreiro y Teberosky, 1981).

Suelen utilizar variadas estrategias de animación y promoción de la lectura para propiciar el desarrollo de un pensamiento crítico reflexivo como objetivo educacional supremo que refleje aprendizajes pertinentes. Recurren a lecturas en clase, discusión alrededor de las ideas propuestas por los autores, recomendaciones de títulos, temas y autores diversos, uso de fichas, guías; utilizan la lectura como vehículo efectivo para promover aprendizajes (Girón Castro, Jiménez Camargo y Lizcano Rivera, 2005; Van Dijk, 1980). Sin embargo, conciben la expresión oral como fórmula general para socializar ideas, compartir saberes, comprobar y valorar la criticidad de los estudiantes (Ong \& Hartley, 2016).

Un hallazgo importante dentro de este proceso asociado a las estrategias utilizadas en el aula está relacionado con la poca o escasa promoción de la escritura como actividad reflexiva del acto lector. Asimismo, no se percibe la concreción dentro de las estrategias 
identificadas, de actividad de creación literaria, producción o análisis textual, recurriendo a la escritura como medio expedito de expresión (Dubois, 2011). Los docentes, en general, se preocupan por que los estudiantes participen efectivamente en la construcción de sus propios aprendizajes, fortaleciendo la autonomía en la manifestación de sus pareceres, puntos de vista, recurriendo generalmente al debate, la discusión, mediando como instrumento detonador, la indagación (Girón Castro, Jiménez Camargo y Lizcano Rivera, 2005; Zuluaga, 1999).

El tercer objetivo de investigación: Determinar cuáles prácticas pedagógicas aportan al desarrollo del pensamiento crítico reflexivo, asociado de manera directa con el eje categorial, Práctica pedagógica. De acuerdo con el análisis realizado a la información obtenida luego de la aplicación de las técnicas de investigación cualitativa, Observación no participante y Entrevista semiestructurada, se puede concluir que las prácticas pedagógicas que propician el desarrollo de un pensamiento reflexivo en los estudiantes están relacionadas con hacer de la lectura un vehículo educativo privilegiado promueve la formación de una conciencia crítica en la que el lenguaje cumple un rol relevante (Saussure, 1959), posibilitando al estudiante indagar, cuestionar y juzgar, recurriendo a la contrastación, a la consulta de otras fuentes para argumentar sus posturas o juicios (Avendaño, 2013; Carlino, 2006, Ong, 2011).

Leer críticamente posibilita encuentros más profundos con la verdad, la realidad y la vida. La expresión oral en el aula promueve el desarrollo de la autoestima, exploración y organización del pensamiento (Rodríguez Castro, 2014). La animación y promoción de la lectura que realizan los docentes se inscriben en la estructura de un plan lector institucional en el que leer, conceptuar, organizar ideas, orientan la formación de un pensamiento autónomo en el que el diálogo establece las normas de participación en la creación de ambientes de aprendizaje (Garavito, 2016; Tovar, 2009).

De manera general, puede concluirse, que la condición de ser lector crítico determina mejores prácticas pedagógicas que orienten a la formación del pensamiento reflexivo en los estudiantes, pues el saber pedagógico, como lo planteaba Zuluaga (1996) debe entenderse como aquello que el docente hace con lo que sabe para promover la enseñanza-aprendizaje. Y esta experiencia de la práctica pedagógica en el aula, demostró que los docentes con hábito, si son críticos, aplican estrategias diversas y significativas para promover el pensamiento reflexivo en sus estudiantes con un nivel de pertinencia y compromiso más elevado que el de las estrategias implementadas por un docente cuyo hábito lector no supera los niveles normales de lectura por placer, entretenimiento o información (Campos, 2007; Cassany, 2013 Moreno, 2005; Munita, 2016).

Los hallazgos evidencian la necesidad de reforzar permanentemente los procesos de animación y promoción de la lectura, involucrando a todos los docentes en prácticas efectivas de lectura, que propicie un nivel de lectura crítico; vincular a estas experiencias a padres de familia y demás miembros de la comunidad educativa para hacer de la lectura y la formación del pensamiento reflexivo una intencionalidad pedagógica institucional (Jones e Idol, 1990; Granado \& Puig, 2014).

Asimismo, la promoción implica el desarrollo de actividades y procesos de escritura creativa a través de talleres, guías y ejercicios que involucren análisis textuales, creación literaria y reflexión escrita sobre la realidad (Tovar, 2009). Es necesario que la lectura crítica sea orientada de manera efectiva, partiendo de una real conceptualización, instrucción y aplicación de técnicas y procedimientos para su desarrollo (Barrios García, 2015; Zuluaga, 1999). Si la lectura crítica hace parte de los hábitos de los maestros, de los estudiantes y de 
la Institución, seguramente el pensamiento reflexivo hallará mejor campo de cultivo para su aplicación y desarrollo (Moreno, 2005; Munita, 2016).

\section{REFERENCIAS BIBLIOGRÁFICAS}

Alderson, J. (2000). Evaluación de la lectura. Cambridge: Cambridge University Press.

Avendaño, G. (2013). ¿Es el buen lector un buen escritor? En Ponencia presentada en el Congreso Internacional Lectura y escritura en la sociedad global. Barranquilla: Universidad del Norte (12).

Barrios García, E. (2015). Amplitud lectora de los docentes de primaria en sonora y su relación con los conocimientos y actitudes hacia el programa nacional de lectura y escritura. (Tesis de maestría). Centro de Investigación en Alimentación y Desarrollo.

Boisvert, J. (2004). Formación del Pensamiento Crítico. México: Fondo de Cultura Económica.

Caldera de Briceño, R., Escalante de Urrecheaga, D. \& Terán de Serrentino, M. (2010). Práctica pedagógica de la lectura y formación docente. Revista de Pedagogía, 31(88), 15-38.

Campos, A. (2007). Pensamiento crítico. Técnicas para su desarrollo. Bogotá: Colección Aula Abierta Magisterio.

Carlino, P. (2006). La escritura en la investigación. Buenos Aires: Universidad de San Andrés.

Cassany, D. (2005). Los significados de la comprensión crítica. Lectura y vida. Barcelona: Universitat Pompeu Fabra.

Cassany, D. (2013). Tras las líneas. Sobre la lectura contemporánea. Barcelona: Anagrama.

Cassany, D. (2016). Aproximaciones a la lectura crítica. Teoría, ejemplos y reflexiones. Barcelona: Universitat Pompeu Fabra.

Castro, C. R. (2014). La literatura en la escuela: una mirada desde los textos, los contextos y el maestro. Nodos y Nudos, 4(36).

Chevallard, Y. (1998). La transposición didáctica. Del saber sabio al saber enseñado. Buenos Aires: Aique.

Chois, P. (2005). Sobre la lectura y la escritura en la escuela ¿qué enseñar? En La didáctica de la lengua materna. Cali: Universidad del Valle.

Dubois, M. E. (2011). La lectura en la formación y actualización del docente. Comentario sobre dos experiencias. Legenda, 15(12).

Eagleton, T. (1998). Una introducción a la teoría literaria. México: Fondo de Cultura Económica.

Ferreiro, E. (1991). La construcción de la escritura en el niño. Lectura y vida, 12(3), 5-14.

Ferreiro, E. \& Teberosky, A. (1981). La comprensión del sistema de escritura: construcciones originales del niño e información específica de los adultos. Lectura y Vida, 2(1), 6-14.

Flórez Ochoa, R. (2013). Estrategias de enseñanza y pedagogía. Actualidades pedagógicas, 1(61), $15-26$.

Freire, P. (1989). La educación como práctica de la libertad. Madrid: Siglo XXI Editores.

Freire, P. (2010). Cartas a quien pretende enseñar. México: Siglo XXI Editores.

Garavito, J. (2016). Encrucijadas pedagógicas: Resignificación, emergencias y praxis educativa. Maracaibo: Universidad del Zulia.

Girón Castro, S; Jiménez Camargo, C. \& Lizcano Rivera, C. (2005). ¿Cómo hacer lectura crítica? Bogotá: Ediciones Universidad Sergio Arboleda.

Giroux, H. (1990). Los profesores como intelectuales. Paidós: Barcelona.

Granado, C. y Puig, M. (2014). ¿Qué leen los futuros maestros y maestras? Un estudio del docente como sujeto lector a través de los títulos de libros que evocan. Ocnos, (11), 93-112.

Guzmán, S. \& Sánchez, P. (2006). Efectos de un programa de capacitación de profesores en el desarrollo de habilidades de pensamiento crítico en estudiantes universitarios en el Sureste de México. Revista electrónica de investigación educativa, 8(2), 1-17. 
Hurtado, J. C. T. (2006). Investigación cualitativa: comprender y actuar. Editorial La Muralla.

Hurtado, J. (2010). El proyecto de investigación comprensión holística de la metodología y la investigación. Caracas: Quirón.

Jones, B. e Idol, L. (1990). Introduction, en B. Jones y L. Idol (eds.), Dimensions of thinking and cognitive instruction. Hillsdale: LEA, 1-11.

Kaufman, A. M. (1994). Escribir en la escuela: qué, cómo y para quién. Lectura y vida, 15(3), 15-32.

Klooster, D. J., Steele, J. L. \& Bloem, P. L. (2001). Ideas without Boundaries: International Education Reform through Reading and Writing for Critical Thinking. Newark: International Reading Association.

Martínez Miguélez, M. (2009). Ciencia y arte en la metodología cualitativa. Métodos hermenéuticos. Métodos fenomenológicos. Métodos etnográficos. México DF, México: Trillas.

Martínez Miguélez, M. (2015). Hermenéutica y análisis del discurso como método de investigación social. Paradigma, 23(1), 9-30.

Medina, A. (2006). Enseñar a leer ya escribir: ¿en qué conceptos fundamentar las prácticas docentes? Psykhe, 15(2), 45-55.

Moreno, V. (2005). Lectores competentes. Revista de Educación, núm. extraordinario, pp. 153-167

Munita, F. (2016). Prácticas didácticas, creencias y hábitos lectores del profesor en una escuela exitosa en la promoción lectora. Ocnos, 15(2), 77-97

Ong, W. (2011). Oralidad y escritura. Tecnologías de la palabra (Traducción de Angélica Scherp). México: Fondo de Cultura Económica

Ong, W. J. \& Hartley, J. (2016). Oralidad y escritura: tecnologías de la palabra. Fondo de Cultura Económica.

Parodi, G. (2003). Relaciones entre lectura y escritura: una perspectiva cognitiva discursiva. Bases teóricas y antecedentes empíricos. Chile: Ediciones Universitarias de Valparaíso.

Quintana, A. y Montgomery, W. (2006). Psicología: Tópicos de actualidad. Lima: UNMSM.

Saiz, C. \& Rivas, S. (2008). Intervenir para transferir en pensamiento crítico. Praxis, 10(13), 129149.

Saussure, F. D. (1959). Course in general linguistics (W. Baskin, Trans.). New York: Philosophical Library.

Tovar, R. M. (2009). La formación como lector y escritor: Un reto para el docente. Educere, 13(44), 109-116.

Van Dijk, T. (1980). Estructuras y Funciones del Discurso. Una Introducción Interdisciplinaria ala Lingüística del Texto y a los Estudios del Discurso. México: Siglo Veintiuno Editores.

Yepes Osorio, L. B. (2001). La promoción de la lectura: conceptos, materiales y autores. Medellín, CO: Comfenalco-Antioquia.

Zuluaga, O. (1996). Una respuesta desde la pedagogía a los interrogantes que la sociedad actual le hace a la escuela. En: Arte Y Conocimiento. Anthropos, Editorial Universidad de Antioquia.

Zuluaga, O. (1999). Pedagogía e historia: la historicidad de la pedagogía, la enseñanza, un objeto de saber. Santafé de Bogotá: Siglo del Hombre Editores. 